\title{
Método Simplificado de Conocimiento en Psicoprofilaxis Obstétrica
}

\author{
Equipos de la Clínica de Maternidad "David Restrepo" de Bogotá \\ e "Iván Pavlov" de Bucaramanga
}

Ponencia presentada al III Simposio Colombiano de Psicoprofilaxia Obstétrica Julio de 1961

\section{INTRODUCCION}

Después de varios años de estar aplicando en Colombia el método Psicoprofiláctico, con ligeras variaciones entre los distintos equipos, pero siguiendo en líneas generales las normas preconizadas por las escuelas europeas, hemos llegado a la conclusión de que hasta ahora solamente se han beneficiado las clases sociales de nivel económico más alto.

Por otra parte, al interrogar a las señoras que han tenido su parto por el método, hemos observado que un número considerable de aquellas que han logrado la indolorización completa del trabajo, posee limitados y aún confusos conocimientos sobre anatomía, mecanismo del parto, mecanismo del sistema nervioso, etc., mientras que pacientes que han asimilado las enseñanzas de estos mismos tópicos, obtienen malos resultados desde el punto de vista de indolorización.

En nuestro interés por encontrar un sistema de educación más económico, de más fácil aplicación, que pueda ser más ampliamente divulgado, y considerando que el fundamento del parto sin dolor, de acuerdo con las teorías de Lamaze, es la creación de reflejos condicionados, hemos pensado en la posibilidad de crear estos mismos reflejos disminuyendo al mínimo las explicaciones teóricas, las cuales por lo demás, si bien son un factor de gran valor para despertar el interés en las pacientes más cultas, en un número considerable de ellas, que poseen escasa cultura, no cumplen esta función. Intentamos por lo tanto crear el reflejo a ba- 
se de la repetición de los estímulos y las respuestas haciendo simulacros de parto después de haber realizado una descripción muy sencilla del mismo, en la cual se insiste en la conducta que deberá seguir la madre en cada una de las fases del trabajo. En esta forma se crearía el reflejo de manera semejante a como se crea en los animales de experimentación y en el aprendizaje de acciones tan complejas como caminar, montar en bicicleta, o en patines. El estímulo será la contracción uterina y en su reemplazo la palabra “contracción”, la respuesta será la actividad cortical desplegada para regular la relajación muscular y la respiración, las cuales se practican en forma idéntica a la acostumbrada en el método clásico.

Creemos que el sistema que proponemos es una contribución útil en el mejoramiento y simplificación de los métodos clásicos de psicoprofilaxia obstétrica y confiamos en que sea acogido con entusiasmo.

\section{MATERIAL Y METODOS}

Intentamos el condicionamiento en un total de 231 pacientes, pertenecientes a dos grupos social, cultural y económicamente distintos: el primero constituído por 105 pacientes de la clínica de Maternidad "David Restrepo" de Bogotá, de clase media económica con un nivel cultural mediano; el segundo formado por 126 pacientes del servicio de caridad del Hospital de "San Juan de Dios" de Bucaramanga, con un nivel económico bajo y aún más bajo nivel cultural.

Dictamos una sola conferencia cuyo esquema es más o menos el siguiente:

Después de unas breves palabras sobre el parto sin dolor que servirán de introducción, decimos que para que se logre es necesario que la paciente se encuentre tranquila, colaborando con el trabajo y no obstaculizándolo, para lo cual se requiere que sepa en qué consiste y qué debe hacer durante el mismo.

Por esto empezamos por decir que el niño se ha desarrollado dentro de la madre, en un órgano denominado matriz o útero, que le podemos comparar con un talego o saco provisto de una jareta que se llama "el cuello" y que éste se encuentra cerrado durante todo el embarazo para impedir que el contenido, es decir el niño, salga de allí antes de tiempo. 
Este talego (la matriz) está formado por un tejido de hilos entrelazados en todas direcciones, como los hilos de una tela cualquiera, pero que están formados por una substancia especial que tiene la propidead de acortarse y alargarse como el caucho, de tal manera que si se acortan todos los hilos al mismo tiempo, el tamaño del talego disminuye en todas direcciones y tratará así de expulsar su contenido. Cada vez que se contraen los hilos, que la matriz se achica en todos los sentidos, decimos que se ha presentado una contracción uterina.

Durante 9 meses, el niño ha venido creciendo y desarrollándose, perfeccionando todos y cada uno de sus órganos, y recibiendo la alimentación de la sangre materna a través del cordón umbilical que lo une a la madre por medio de la placenta, hasta que llega un momento en el cual ya está suficientemente desarrollado como para vivir en el exterior y defenderse por sí solo; entonces debe salir de allí, debe nacer, debe presentarse el parto.

Después les explicamos en una forma sencilla cómo sale el niño del interior de la matriz atravesando el canal que une a ésta con la piel, con el exterior (la vagina) y comenzar a vivir por sus propios medios.

Les recalcamos que la matriz es un talego que tiene una boca, que se encuentra cerrada durante todo el embarazo por una jareta y que esa jareta la hemos llamado cuello. Les decimos que para sacar lo que está adentro debemos primero abrir la puerta, abrir el cuello, lo cual se llama en lenguaje más técnico "dilatar el cuello", para que pueda pasar a través de él el niño. Que ésto se logra por la propiedad que tienen las paredes de la matriz de acortarse, lo mismo claro está, que los hilos que las forman. Al acortarse halan de los bordes del cuello, luego se alargan y descansan; a los pocos minutos se acortan nuevamente y así, poco a poco, lentamente lo van abriendo, es decir, dilatándolo. Cada vez que esto sucede, el niño es empujado hacia afuera pero no podrá salir de allí hasta tanto no se "abra completamente la puerta".

Una vez terminada la dilatación del cuello pasará la cabeza a través del mismo y comenzará a atravesar ese canal delgado que une la matriz con el exterior, la vagina al cual también tiene que ir ensanchando poco a poco para dar paso a la cabeza del niño. Este trayecto es muy corto y su recorrido se hace relativamente rápido, generalmente menos de una hora en las señoras 
que van a tener el primer hijo y mucho menos en las multíparas. Salida al exterior la cabeza, el resto del cuerpo la sigue con gran facilidad y rapidez y unos cinco o diez minutos después saldrá también la placenta.

A continuación les decimos que este proceso, este parto que acabamos de describir es un proceso generalmente doloroso, no sabemos desde cuándo ni si hubo una época en el comienzo de la humanidad en que no lo fué, etc., etc., pero que afortunadamente existe un procedimiento, ya bien estudiado y experimentado, que permite hacer que se obtenga el parto sin dolor.

En seguida les advertimos que con el entrenamiento que van a recibir, siempre y cuando lo practiquen todos los días, pensando todas las veces que están en trabajo de parto y poniendo toda la atención e interés, se llegará a la indolorización por mecanismo suficientemente estudiados y demostrados, cuyo conocimiento por parte de ellas no es necesario para obtener buenos resultados, así como nadie requirió conocimientos de anatomía y fisiología del sistema nervioso para aprender a caminar.

Después les damos una breve explicación sobre las contracciones del embarazo y la sensación que se experimenta, advirtiéndoles que estas contracciones son de entrenamiento de la matriz para cuando llegue el momento de efectuar el trabajo verdadero; son irregulares y de corta duración. Les agregamos que cuando se desprende el tapón mucoso y cuando las contracciones se hacen regulares y frecuentes se está iniciando el trabajo del parto.

A continuación se les enseña la conducta que deben adoptar durante el trabajo: que durante las primeras contracciones, cuando se encuentran espaciadas más o menos cada 15 minutos bastará con relajar los músculos completamente, como van a aprender en las sesiones prácticas. Que cuando estén más frecuentes, cada 5 o 7 minutos, y duren más tiempo, 30, 40 segundos, a más de la relajación practicarán un tipo de respiración: la respiración profunda.

Se les dice que en un período más avanzado del parto, el médico les ordenará practicar la respiración superficial y acelerada, por último la superficial acelerada y soplada, y el pujo.

Hasta aquí sería la conferencia que se adicionará con los dibujos y proyecciones que se consideren necesarios. 
Se les harán luego cuatro clases prácticas o entrenamientos, una semanal, de una duración aproximada de una hora. En la primera se les enseñará la relajación, los tres tipos de respiración y el pujo, sin pretender que lo pueden hacer perfectamente en esta sesión.

En las sesiones siguientes se hará un simulacro de parto, empezando con la relajación muscular solamente, durante unas 4. o 5 contracciones, luego relajación y respiración profunda y lenta por otras cuantas contracciones; más tarde respiración superficial acelerada y soplada con relajación, y finalmente pujo y respiración jadeante hasta que nazca el niño.

Durante esos simulacros de parto se les dirigirá más o menos en la siguiente forma: Va a empezar la contracción, lista, está empezando la contracción, se va a relajar completamente, van a aflojar sus músculos al tiempo con la contracción, están sintiendo como se pone dura la matriz, está aumentando la fuerza de la contracción, relájense, respiren... está disminuyendo la fuerza... está pasando... pasó ya la contracción, descansen... Se continuará así la dirección del trabajo hasta que "nazca el niño". En el curso de cada entrenamiento se irá corrigiendo todo defecto en la relajación, respiración, etc.

Finalmente insistimos en que estos ensayos de parto deberán ser repetidos varias veces en su casa, en donde irán a tener tres, cuatro o más partos por día.

La valoración de resultados se ha hecho de acuerdo con el sistema de calificación aprobado en Medellín, en el II Simposio de Psicoprofilaxia Obstétrica de 1960, calificación que se ha aplicado con rigor absoluto.

\section{RESULTADOS}

Los resultados de la Clínica de Maternidad "David Restrepo" de Bogotá se resumen en los siguientes cuadros:

\section{CUADRO $N^{\circ} 1$}

CLINICA "DAVID RESTREPO"

$\begin{array}{lcccc}\text { Paridad } & \text { Buenos } & \text { Regulares } & \text { Malos } & \text { Total } \\ \text { Primíparas } & 13 & 6 & 11 & 30 \\ \text { Multíparas } & 31 & 23 & 21 & 75 \\ \text { Totales } & 44 & 29 & 32 & 105\end{array}$




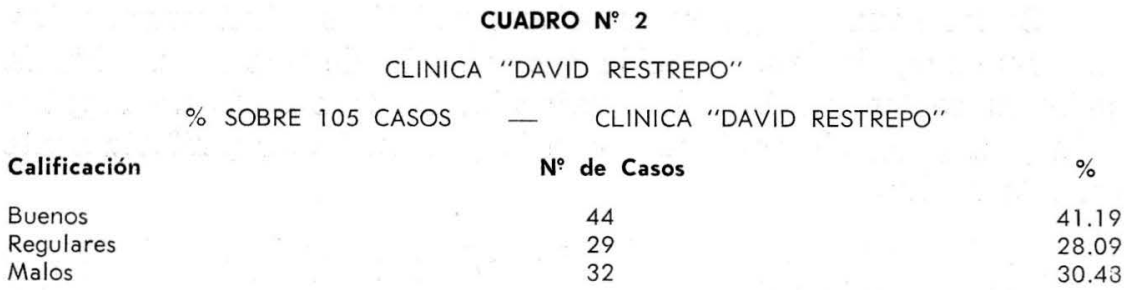

Calificación

Buenos

Regulares

Malos

№ de Casos

44

29

32

$\%$

41.19

28.09

30.43

CUADRO N: 3

CLINICA "DAVID RESTREPO"

Presentación y Variedad de Pos.

Pos. Bueno
18
13
7
3
1
1
2

\section{Regulares}

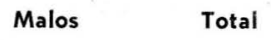

O. I. A.

O. D. P

O. D. A.

O. I. P.

S. I. P.

O. D. T

O. I. T.

13
7
3
1
1
2
2

$\begin{array}{rr}11 & 42 \\ 10 & 30 \\ 8 & 18 \\ 2 & 6 \\ - & 1 \\ -1 & 4 \\ - & 4\end{array}$

\section{CUADRO N 4 \\ CLINICA "DAVID RESTREPO"}

\section{Clasificación}

\section{Buenos}

Regulares

Malos

\begin{tabular}{cc}
\multicolumn{2}{|c}{ Método Clásico } \\
No de Casos & $\%$ \\
263 & 41.02 \\
164 & 25.58 \\
214 & 33.38
\end{tabular}

Método de Conf. Unica No de Casos

41.19

28.09 30.48

En este cuadro se comparan los resultados obtenidos en la Clínica David Restrepo en 641 casos atendidos de Febrero 13 de 1959 a Abril 30 de 1960, por el método Clásico con 105 casos atendidos en la misma Clínica de Abril de 1960 a Mayo de 1961 por el método de Conferencia Unica.

\section{CUADRO № 5}

CLINICA "DAVID RESTREPO"

EVALUACION DEL RECIEN NACIDO

$\begin{array}{cc}\text { Apgar } & \text { No de Casos } \\ 10 & 101 \\ 9 & 1 \\ 8 & 2 \\ 7 & 1\end{array}$

Los resultados obtenidos en el "Hospital San Juan de Dios" de Bucaramanga, se resumen en los cuadros siguientes: 
CUADRO N: 1

"HOSPITAL DE SAN JUAN DE DIOS"

$\begin{array}{lcccc}\text { Paridad } & \text { Buenos } & \text { Regulares } & \text { Malos } & \text { Total } \\ \text { Primíparas } & 14 & 19 & 8 & 41 \\ \text { Multíparas } & 54 & 29 & 2 & 85 \\ \text { Totales } & 68 & 48 & 10 & 126\end{array}$

Por este cuadro se puede concluír que el mayor número de casos buenos corresponde a las multíparas; los casos regulares y malos son más numerosos entre las primíparas.

\section{CUADRO N: 2}

"HOSPITAL DE SAN JUAN DE DIOS"

$\begin{array}{ccccc}\text { Presentación y Variedad de Pos. } & \text { Buenos } & \text { Regulares } & \text { Malos } & \text { Total } \\ \text { O. I. A. } & 42 & 30 & 5 & 77 \\ \text { O. D. P. } & 15 & 8 & 3 & 26 \\ \text { O. D. A. } & 6 & 8 & 2 & 16 \\ \text { O. I. P. } & 4 & 1 & - & 5 \\ \text { S. I. A. } & 1 & - & - & 1 \\ \text { A. I. D. P. } & 1 & - & - & 1\end{array}$

En resumen podemos concluír que la presentación y la variedad de posición no modificaron los resultados.

CUADRO N: 3

"HOSPITAL DE SAN JUAN DE DIOS"

GRUPO ESPECIAL

$\begin{array}{lcccc}\text { Intervenciones } & \text { Buenos } & \text { Regulares } & \text { Malos } & \text { Total } \\ \text { Fórceps } & 2 & - & - & 2 \\ \text { Cesáreas } & 1 & 2 & - & 3 \\ \text { Ventosa Obstétrica } & 1 & 3 & - & 4\end{array}$

En las aplicaciones de fórceps y de Ventosa Obstétrica de Malmstrom, no tuvimos necesidad de usar anestesia. Los casos que terminaron en operación cesárea se calificaron hasta el momento en que se indicó la intervención y usamos anestesia raquídea.

\section{CUADRO N: 4}

"HOSPITAL DE SAN JUAN DE DIOS"

EVALUACION DEL RECIEN NACIDO

$\begin{array}{cc}\text { Apgar } & \mathbf{N} \div \text { de Casos } \\ 10 & 110 \\ 9 & 12 \\ 8 & 2 \\ 7 & 2\end{array}$

Como vemos es evidente que en la inmensa mayoría de casos el estado físico del recién nacido es excelente. 


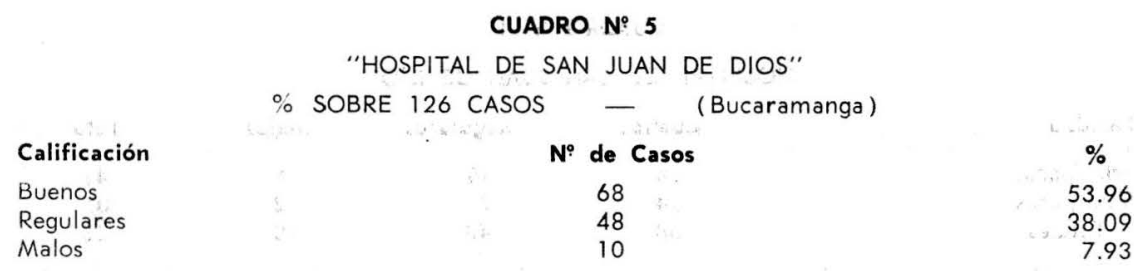

Este cuadro demuestra la eficiencia del método "de conferencia única".

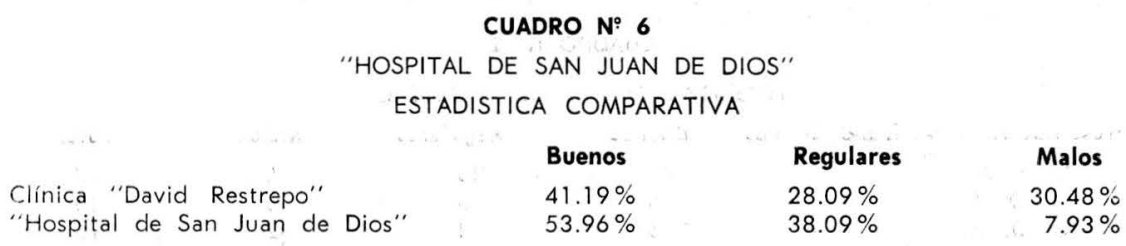

\section{Regulares}

$28.09 \%$ $38.09 \%$

Malos

$30.48 \%$ $7.93 \%$

Los mejores resultados obtenidos en el grupo de Bucaramanga se podrían explicar porque este tipo de pacientes tiene un umbral al dolor más elevado.

\section{CUADRO N: 7}

CLINICA "DAVID RESTREPO" $Y$ "HOSPITAL DE SAN JUAN DE DIOS" RESUMEN GENERAL DE RESULTTADOS

$\begin{array}{lrr}\text { Calificación } & \text { No de Casos } & \% \\ \text { Buenos } & 112 & 48.48 \\ \text { Regulares } & 77 & 33.33 \\ \text { Malos } & 42 & 18.18 \\ \quad \text { Total } & 231 & 99.99\end{array}$

Los resultados del total de los casos estudiados en este trabajo demuestran una vez más la posibilidad de aplicar con éxito el método propuesto.

\section{CONCLUSIONES}

1 - El "método de Conferencia Unica" produce resultados sensiblemente iguales a los obtenidos por el "Método Clásico".

2 - Los resultados obtenidos por el "Método de Conferencia Unica" nos autorizarían para aconsejar su empleo en la preparación de pacientes especialmente del tipo hospitalario, de escasa cultura, y para que quienes el tiempo empleado en la asistencia a cuatro o más conferencias representa, a más de un esfuerzo considerable, una disminución en las bajas entradas famiiiares. 